\title{
Statistical analysis of the associations between polymorphisms within aldehyde dehydrogenase $2(A L D H 2)$, and quantitative and qualitative traits extracted from a large-scale database of Japanese single-nucleotide polymorphisms (SNPs)
}

\author{
Junichiro Nose $\cdot$ Akira Saito $\cdot$ Naoyuki Kamatani
}

Received: 15 December 2007 / Accepted: 30 January 2008/Published online: 4 March 2008

(C) The Japan Society of Human Genetics and Springer 2008

\begin{abstract}
A scan of 4,190 single-nucleotide polymorphisms (SNPs) in 199 different genes using 38 different quantitative traits to search for associations between genotypes and phenotypes detected an association between the genotypes at rs671 of $A L D H 2$ and gamma-glutamyltranspeptidase (gamma-GTP) levels. We examined the associations between five factors such as gender, age, rs671 genotype, alcohol-drinking habit, and serum gamma-GTP level and found that all pairs were associated except for the pair of rs671 genotype and gender and rs671 genotype and age. We further analyzed the data by both multiple regression and subgroup analyses and found that the associations between rs671 genotype and alcohol-drinking habit, alcohol-drinking habit and gamma-GTP level, gender and gamma-GTP, and age and gamma-GTP were independent of other factors. Conversely, the association between rs671 genotype and gamma-GTP level was dependent on alcohol-drinking habit. Associations between genetic and environmental factors will become a focus of medical and biological studies. Our study has shown that (1) a large sample size combined with a replication study is necessary to overcome the multiple-comparison problem, and (2) subgroup analysis along with logistic and linear multiple regression analysis may be useful to dissect a complicated relationship.
\end{abstract}

\footnotetext{
J. Nose $\cdot$ A. Saito $\cdot$ N. Kamatani $(\square)$

Division of Genomic Medicine, Department of Advanced

Biomedical Engineering and Science and Institute

of Rheumatology, Tokyo Women's Medical University,

10-22 Kawada-cho, Shinjuku-ku, Tokyo 162-0054, Japan

e-mail: kamatani@ior.twmu.ac.jp
}

Keywords Alcohol - Multiple comparison · Multiple regression analysis - Association study · Pharmacogenomics · Pharmacogenetics

\section{Introduction}

Due to the availability of accurate genotyping systems for thousands or millions of single-nucleotide polymorphisms (SNPs), genetic association studies are now a focus of medical research (Kamatani et al. 2004; Rosalki 1973, 1974; Boone et al. 1977; Spencer-Peet et al. 1975; Teschke et al. 1977; Impraim et al. 1982). One problem in using thousands of SNPs is multiple comparisons. Thus, if the test is performed using thousands of SNPs, a certain number of SNPs show very low $P$ values, even if they are not truly associated with the traits. To overcome the problem of multiple testing, such methods as Bonferroni's correction are used; however, in this case, extremely low $P$ values are required for SNPs to be truly associated with the traits.

Another problem in association studies for multifactorial diseases is relatively small effect sizes. Unlike Mendelian traits, the effects of genotypes on phenotypes are not large in such diseases. To cope with the problem of small effect size as well as multiple comparisons, a large sample size is necessary.

An additional issue to consider in the study of multifactorial traits is the effect of nongenetic factors. For example, gender, age, habit, diet, and exposure to chemicals may be associated with the development of phenotypes. In some cases, such nongenetic factors may affect each other or may interact with genetic factors for the development of phenotypes leading to a very complicated structure of interactions. In this investigation, we show 
such an example and a potential method to dissect the complicated structure of the associations.

Enzymes and transporters possibly related to transportation, metabolism, and action of chemical agents may be associated with either efficacy or adverse drug interactions. In a previous study, we constructed a database of SNPs and haplotypes for 4,190 SNPs in 199 different genes using blood samples from 752 Japanese subjects (Kamatani et al. 2004). For those subjects, 38 different quantitative traits were available. Some of these traits may be associated with either genotypes or diplotype configurations (combination of two haplotypes) in some genes independently or with interactions. We performed an extensive analysis to search for such associations using all available genomic variations and traits. Through such an analysis, we observed a strong association between SNPs of aldehyde dehydrogenase 2 (ALDH2) and serum gamma-glutamyltranspeptidase (gamma-GTP) levels. Serum gamma-GTP elevates after alcohol ingestion (Rosalki 1973, 1974; Boone et al. 1977; Spencer-Peet et al. 1975; Teschke et al. 1977), and a polymorphism (rs671) in $A L D H 2$ is associated with individual tolerance to alcohol (Impraim et al. 1982; Goedde et al. 1986, 1992; Crabb et al. 1989; Shibuya et al. 1989). In fact, gamma-GTP levels were associated with alcohol-drinking habit and gender in addition to the rs671 SNP genotype in our data. In this study, we analyzed data to dissect the complicated structure of associations between genomic variations in $A L D H 2$, alcohol-drinking habit, gender, age, and serum gamma-GTP levels to establish an effective method to investigate associations of genomic variations with both qualitative and quantitative traits.

\section{Materials and methods}

\section{Subjects}

New samples were not collected; data collected in a previous study (Kamatani et al. 2004) were used. From that study, genotype data of 4,190 SNPs in 199 different genes from 752 Japanese subjects were available. Those SNPs had been found from genes coding for enzymes and transporters possibly related to transportation, metabolism, and action of chemical agents (Kamatani et al. 2004). SNPs within the same genes are often in linkage disequilibrium (LD) with each other, and the results of the LD analysis have been described elsewhere (Kamatani et al. 2004). For those subjects, 38 different quantitative traits including serum gamma-GTP levels were obtained. Informed consents had been obtained from all subjects for using their DNA for any association studies, and the study had been approved by the institutional ethical committee.
Genotyping

Genotyping was performed by the Invader system (Ohnishi et al. 2001). Methods for the genotype data collection have been described previously (Kamatani et al. 2004).

Scan of the associations between SNPs and quantitative phenotypes

The computer program to scan all 4,190 SNPs and 38 different quantitative traits to find associations was made with C language. All other calculations and statistical analyses were performed in $\mathrm{R}$.

\section{SNP information}

SNP information such as order and map position was obtained from the National Center for Biotechnology Information (NCBI) database (http://www.ncbi.nlm.nih. gov/). LD parameters $D^{\prime}$ and $r^{2}$ were calculated using PENHAPLO and QTLHAPLO programs (Ito et al. 2004; Shibata et al. 2004).

Statistical analysis

Association between genotypes and phenotypes was performed by the single-locus analysis only. No haplotypebased association tests were performed. Since most of the 38 quantitative variables were not normally distributed, nonparametric methods were used. To compare quantitative variables in two different groups, the Mann-Whitney $U$ test was used. To compare the same variables in more than two different groups, the Kruskal-Wallis test was used. To test the correlation between two quantitative variables, Spearman's rank correlation coefficient was used. When the correlation between age and gamma-GTP level was examined, the parametric method after the logarithmic conversion of gamma-GTP level was used. All statistical tests and inferences, including logistic multiple regression analysis and linear multiple regression analysis, were performed in the $\mathrm{R}$ environment.

\section{Results}

Scan of associations between genotypes and quantitative traits

The test of association using each of the 4,190 SNPs and each of the 38 different quantitative traits was performed using the Mann-Whitneys test. For each SNP, two different comparisons were made: between one homozygote type and the rest of the subjects and between the other homozygote 
type and the rest of the subjects. When the SNP consisted of $A$ and $G$ alleles, for example, the subjects with the AA genotype were compared with those with the AG or GG genotype for one comparison, whereas the subjects with GG were compared with those with the AA or AG genotype for the other.

It was found that $P$ values were among the lowest for the association between rs4646776 and rs671 SNPs within $A L D H 2$ and serum gamma-GTP levels (Table 1). For rs671, the subjects with the GG genotype [ $n=436$, median 24.5, mean 39.8, standard deviation (SD) 42.0] had significantly higher gamma-GTP levels than those with the AA or AG genotype $(n=314$, median 21.0, mean 31.2, SD 31.4) $\quad(P=0.000299$, Mann-Whitneys $U$ test $)$ (Table 2). The comparison between subjects with the AA genotype and those with the AG or GG genotype (AG + GG) at the same SNP showed a higher $P$ value of 0.0493 (Table 2).

Linkage disequilibrium analysis of rs671 SNP

Closely located SNPs are often in strong linkage disequilibrium with each other. We determined the LD parameters between pairs of nine SNPs including rs671, which is the critical SNP for alcohol intolerance (Impraim et al. 1982; Goedde et al. 1986, 1992; Crabb et al. 1989; Shibuya et al. 1989). Table 1 shows that $D^{\prime}$ values between rs671 and each of the other eight SNPs were either 1.0 or very close to 1 , thereby indicating that rs671 was in almost complete LD (Feldman et al. 1974) with these SNPs. On the other hand, $r^{2}$ values between rs671 and rs4646776 but not between the other SNPs were close to 1.0. These data indicate that rs671 is almost in absolute LD (Walter and Cox 1988; Kasperczyk et al. 1990; Chehab et al. 1991) with rs4646776 but not with the other SNPs. The physical distance between rs671 and rs4646776 is about $11.7 \mathrm{~kb}$, and it is not surprising that the two SNPs in this distance are in absolute LD.

If two SNPs are in absolute LD, it is expected that one of them can represent the other almost completely. As expected, rs4646776 but not the other SNPs was strongly associated with gamma-GTP level (Table 1). When gamma-GTP levels were compared between different genotypes at rs4646776, the difference was statistically significant by the Kruskal-Wallis test $(P=0.00105)$ (Table 1). When gamma-GTP levels were compared between the genotypes $\mathrm{CC}+\mathrm{CG}$ and GG, they were significantly different $(P=0.000321$, Mann-Whitney test).

Association between the genotype at rs671 and gamma-GTP level

SNP rs671 has been known to affect $A L D H 2$ enzyme function (Impraim et al. 1982). Subjects with the AG genotype have a higher sensitivity to alcohol than those with the GG genotype, whereas those with the AA genotype are even more sensitive than those with the AG genotype (Impraim et al. 1982; Goedde et al. 1986, 1992; Crabb et al. 1989; Shibuya et al. 1989).

Although the $P$ value of 0.000299 is quite low compared with the regular significance level of 0.05 , it is much higher than the significance level required after Bonferroni's correction for multiple comparisons. Since the test was performed for 4,190 SNPs and 38 quantitative traits with two different modes, the significance level required after Bonferroni's correction will be $0.05 /(4,190 \times 38 \times 2)=$ $1.57 \times 10^{-7}$.

Next, gamma-GTP levels were compared between three different genotypes at rs671 SNP. Information was

Table 1 Single-nucleotide polymorphisms (SNPs) in $A L D H 2$ gene and linkage disequilibrium (LD) parameters

\begin{tabular}{|c|c|c|c|c|c|c|}
\hline SNP & $\mathrm{MAF}^{\mathrm{a}}$ & Position $^{\mathrm{b}}$ & $D^{\prime}$ & $r^{2}$ & $P$ value $(\mathrm{KW})^{\mathrm{c}}$ & $P$ value (MW) ${ }^{\mathrm{d}}$ \\
\hline rs886205 & 0.198 & 110688810 & 0.965 & 0.0735 & 0.962 & 0.784 \\
\hline rs 440 & 0.162 & 110713097 & 1 & 0.0618 & 0.697 & 0.252 \\
\hline rs441 & 0.161 & 110713232 & 1 & 0.0614 & 0.376 & 0.22 \\
\hline rs4646776 & 0.243 & 110714402 & 0.996 & 0.993 & 0.00105 & 0.000321 \\
\hline rs4646777 & 0.161 & 110714419 & 1 & 0.0613 & 0.448 & 0.268 \\
\hline rs4646778 & 0.227 & 110720166 & 1 & 0.061 & 0.408 & 0.242 \\
\hline rs4646779 & 0.014 & 110722364 & 1 & 0.0045 & - & 0.65 \\
\hline rs671 & 0.243 & 110726149 & - & - & 0.00131 & 0.000401 \\
\hline rs4646782 & 0.376 & 110732652 & 1 & 0.193 & 0.838 & 0.563 \\
\hline
\end{tabular}

Linkage disequilibrium parameters were estimated between rs671 and the other SNPs

${ }^{\text {a }}$ Minor allele frequency estimated by the present data

b Nucleotide position (bp) from contiq accession NT 009775.16

${ }^{c}$ Kruskal-Wallis test for the difference in gamma-glutamyltranspeptidase (gamma-GTP) levels between three different genotypes at rs671

${ }^{\mathrm{d}}$ Mann-Whitneys test for the difference in gamma-GTP levels between the major allele homozygotes and the other genotypes 
Table 2 Comparison of gamma-glutamyltranspeptidase (gamma-GTP) levels between subjects with different genotypes at rs671

\begin{tabular}{|c|c|c|c|c|c|c|}
\hline & \multirow[t]{2}{*}{ Number } & \multirow[t]{2}{*}{ Median } & \multirow[t]{2}{*}{ Mean } & \multirow[t]{2}{*}{ SD } & \multicolumn{2}{|l|}{ Statistical analysis } \\
\hline & & & & & Test & $P$ value \\
\hline Total & 750 & 23 & 36.2 & 38.2 & & \\
\hline AA & 50 & 19.5 & 23.9 & 14.5 & & \\
\hline AG & 264 & 21 & 32.6 & 33.5 & Kruskal-Wallis ${ }^{\mathrm{a}}$ & 0.000988 \\
\hline GG & 436 & 24.5 & 39.8 & 42 & & \\
\hline $\mathrm{AA}+\mathrm{AG}$ & 314 & 21 & 31.2 & 31.4 & MW (vs. GG) ${ }^{\mathrm{b}}$ & 0.000299 \\
\hline $\mathrm{AG}+\mathrm{GG}$ & 700 & 23 & 37.1 & 39.2 & $\mathrm{MW}\left(\right.$ vs. AA) ${ }^{\mathrm{c}}$ & 0.0493 \\
\hline \multicolumn{7}{|l|}{ Male } \\
\hline Subtotal & 447 & 35 & 48.3 & 44.7 & & \\
\hline AA & 35 & 24 & 27.6 & 15.7 & & \\
\hline $\mathrm{AG}$ & 161 & 32 & 43.5 & 38.7 & Kruskal-Wallis ${ }^{\mathrm{a}}$ & $3.02 \times 10^{-5}$ \\
\hline GG & 251 & 39 & 54.4 & 49.7 & & \\
\hline $\mathrm{AA}+\mathrm{AG}$ & 196 & 29 & 40.7 & 36.1 & MW (vs. GG) ${ }^{\mathrm{b}}$ & 0.000146 \\
\hline $\mathrm{AG}+\mathrm{GG}$ & 412 & 36.5 & 50.2 & 46 & $\mathrm{MW}\left(\right.$ vs. AA) ${ }^{\mathrm{c}}$ & 0.000275 \\
\hline \multicolumn{7}{|l|}{ Female } \\
\hline Subtotal & 303 & 16 & 18.3 & 10.6 & & \\
\hline AA & 15 & 14 & 15.1 & 4.22 & & \\
\hline AG & 103 & 14 & 15.7 & 8.16 & Kruskal-Wallis $^{\mathrm{a}}$ & $5.74 \times 10^{-5}$ \\
\hline GG & 185 & 17 & 20 & 11.8 & & \\
\hline $\mathrm{AA}+\mathrm{AG}$ & 118 & 14 & 15.6 & 7.76 & MW (vs. GG) ${ }^{\mathrm{b}}$ & $1.05 \times 10^{-5}$ \\
\hline $\mathrm{AG}+\mathrm{GG}$ & 288 & 16 & 18.4 & 10.8 & $\mathrm{MW}\left(\right.$ vs. AA) ${ }^{\mathrm{c}}$ & 0.3446 \\
\hline
\end{tabular}

${ }^{a}$ Difference in gamma-GTP levels was tested between different genotypes (AA, AG, GG) by Kruskal-Wallis test

b Difference in gamma-GTP levels was tested by Mann-Whitney $U$ test between two groups, one composed of genotype GG and the other composed of the combination of two genotypes AA and AG

${ }^{c}$ Difference in gamma-GTP levels was tested by Mann-Whitney $U$ test between two groups, one composed of genotype AA and the other composed of the combination of two genotypes GG and AG

available for 750 subjects. Comparison between the gamma-GTP levels of the subjects with AA $(n=50)$, AG $(n=264)$, and GG $(n=436)$ genotypes at rs671 are shown by box plots in Fig. 1. Medians, means, and standard deviations (SD) of gamma-GTP levels with the three different genotypes as well as the combined genotypes $(\mathrm{AA}+\mathrm{AG}$ and $\mathrm{AG}+\mathrm{GG})$ are shown in Table 2. The gamma-GTP levels in the three genotypes AA, AG, and GG were significantly different when tested by the Kruskal-Wallis test $(P=0.000988$, Table 2$)$, but the $P$ value was still insufficient to reach the threshold of significance after Bonferroni's correction $\left(3.14 \times 10^{-7}\right)$. Data in Table 2 and Fig. 1 indicate that there is a gene dosage effect in the association between the rs671 SNP and gamma-GTP levels; i.e., this quantitative trait increases with an increasing dose of the $G$ allele.

Association between each pair of five factors

Although the association between the rs671 genotype and gamma-GTP level was extracted from the association study

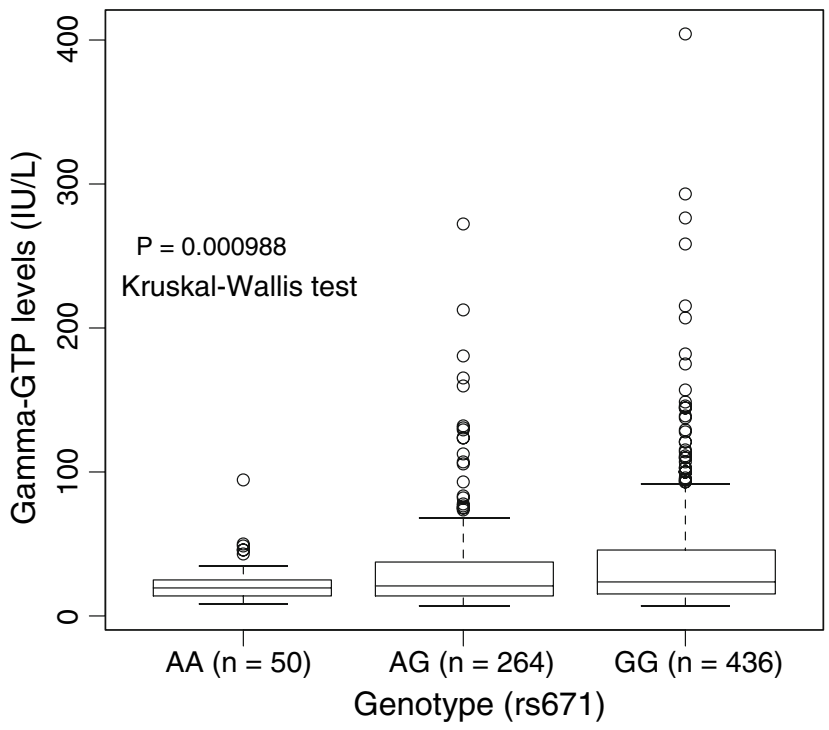

Fig. 1 Comparison of gamma-glutamyltranspeptidase (gamma-GTP) levels between subjects with AA, AG, and $=G G$ genotypes at rs671 are shown as box plots. Male and female subjects were combined as a single sample 
in which multiple SNPs and phenotypes were included, the precise relationship between various factors should be analyzed with care. Previous studies have indicated the cause-and-effect relationship between rs671 genotype and alcohol-drinking habit (Impraim et al. 1982) and between alcohol-drinking habit and gamma-GTP level (Boone et al. 1977). However, other factors, including gender and age, are also likely to be involved.

In this study, we examined the structure of the relationship between two qualitative variables (gender and alcohol-drinking habit), two quantitative variables (age and gamma-GTP level), and the rs671 genotype (Fig. 2). Gender, alcohol-drinking habit, and the rs671 genotype can be interpreted as discrete random variables, whereas both age and gamma-GTP level can be interpreted as continuous random variables. Neither gamma-GTP level nor age is normally distributed, and the application of parametric methods is invalid. We first examined the association between each pair of five factors in Fig. 2. We had already obtained evidence of the association between the rs671 genotype (i.e., number of $\mathrm{G}$ alleles in the genotype) and gamma-GTP level (Figs. 1, 2a; Table 2) by nonparametric methods.

We next examined the association between gender and gamma-GTP level (Fig. 2b). Male subjects had significantly higher gamma-GTP levels than female subjects (Fig. 3a) $\left(P<2.2 \times 10^{-16}\right.$, Mann-Whitney test). We then

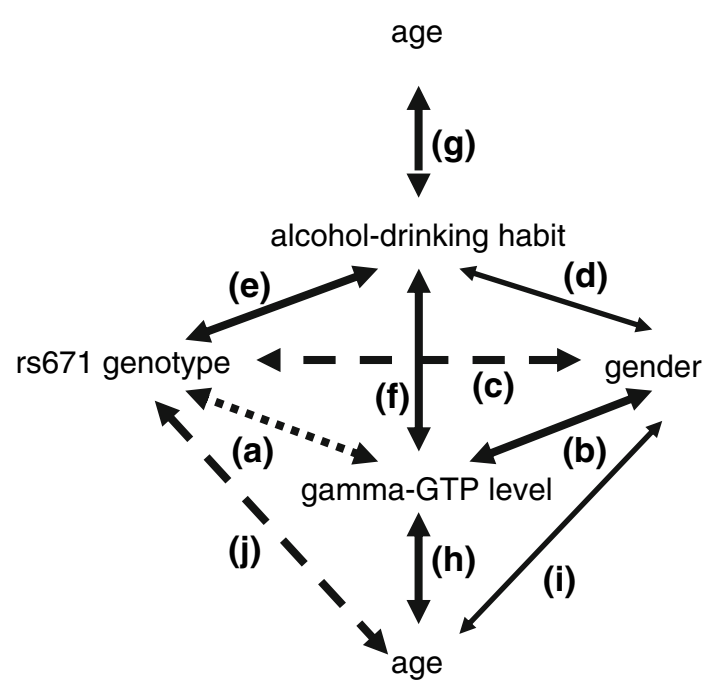

Fig. 2 Relationships between pairs of five different factors: rs671 genotype, gamma-glutamyltranspeptidase (gamma-GTP) level, age, gender, and alcohol-drinking habit. Since there are ten different pairs of factors, we can consider ten different relationships. Except for the relationships between rs671 genotype and gender $(c)$, and age and rs671 genotype $(j)$, all eight other relationships were shown to be dependent. Dependent and independent relationships are expressed by closed and dotted lines, respectively. Relationship between rs671 genotype and gamma-GTP level (a) was apparently dependent but judged to be independent by further analysis examined the association between gender and rs671 genotype (Fig. 2c). Table 3 shows the number of genotypes in male and female subjects. There was no association between gender and rs671 genotype (chi-square test with $2 d f, P=0.202)$. Therefore, as expected from inheritance laws, the rs671 genotype was independent of gender (Fig. 2c).

We then examined the association between gender and alcohol-drinking habit (Fig. 2d). The subjects were categorized based on their answers to the question "Do you drink alcohol"? The three possible answers were 0 "no"; 1 "yes, periodically"; and 2 "yes, almost every day". The question was answered by 734 subjects. Table 4 shows the subjects categorized by gender and their answers to the question. Gender was strongly associated with alcoholdrinking habit (Table 4$)\left(P=1.83 \times 10^{-12}\right.$, chi-square test with $2 d f$ ). Males were categorized as everyday drinkers more frequently than were female subjects [risk ratio (RR) 2.03 , 95\% confidence interval (CI) 1.64-2.50].

We then examined the association between alcoholdrinking habit and the rs671 genotype (Fig. 2e). The values for both variables were available from 732 subjects. Table 5 shows the association between the habit of alcohol ingestion and rs671 genotypes by a $3 \times 3$ contingency table. The proportions of subjects who drank alcohol (periodically or almost everyday) increased significantly with increasing numbers of $G$ allele in the genotype (Table 5) $\left(P<2.2 \times 10^{-16}\right.$, chi-square test with $\left.2 d f\right)$. When the risk of everyday drinking was compared between different rs671 genotypes, the probability of becoming everyday drinkers was 4.69-fold (RR 95\% CI 2.47-8.88, $P=1.22 \times 10^{-15}$, chi-square test with $1 d f$ ) as high for subjects with the AG genotype as those with the AA genotype, whereas it was 5.36-fold (RR 95\%CI 2.84-10.1, $P=5.18 \times 10^{-30}$, chi-square test with $1 d f$ ) as high as those with the GG genotype (calculated from Table 5).

Then, we examined the association between alcoholdrinking habit and gamma-GTP level (Fig. 2f) from 734 subjects. Gamma-GTP levels were much higher in everyday drinkers than in periodic drinkers, and they were much higher in periodic drinkers than in nondrinkers (Fig. 4a) $\left(P<2.2 \times 10^{-16}\right.$, Kruskal-Wallis test $)$. These results indicate that the alcohol-drinking habit was strongly associated with gamma-GTP level (Fig. 2f).

We then examined the association between age and alcohol-drinking habit. Ages of everyday drinkers (mean 42.1, SD 11.1, $n=312$ ) were higher than those of nondrinkers (mean 37.4, SD 11.8, $n=166, P=2.80 \times 10^{-6}$, Mann-Whitney $U$ test) and those of periodic drinkers (mean 35.6, SD 10.8, $n=256, P=6.55 \times 10^{-13}$, MannWhitney $U$ test) (Kruskal-Wallis test, $P=6.66 \times 10^{-13}$ ). These results indicate that age is clearly associated with alcohol-drinking habit (Fig. 2g). In addition, age was 
Fig. 3 a Comparison of gamma-glutamyltranspeptidase (gamma-GTP) levels between male and female subjects. b Comparison of gamma-GTP levels between male and female subjects in nondrinkers.

c Comparison of gamma-GTP levels between male and female subjects in periodic drinkers. d Comparison of gamma-GTP levels between male and female subjects in everyday drinkers
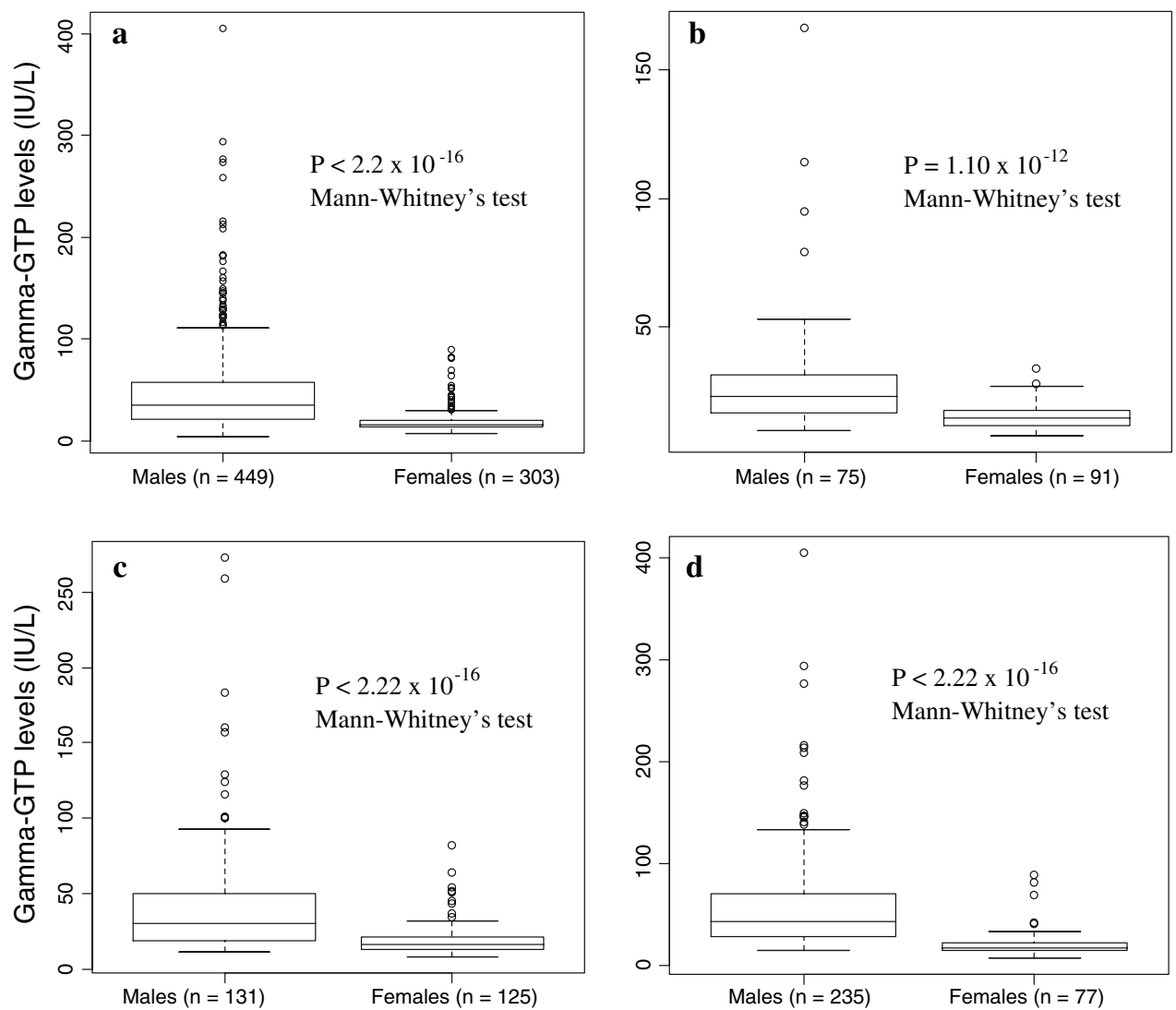

Table 3 Numbers of male and female subjects with different genotypes at rs671

\begin{tabular}{lccc}
\hline Genotype & Male & Female & Total \\
\hline AA & 35 & 15 & 50 \\
AG & 161 & 103 & 264 \\
GG & 251 & 185 & 436 \\
Total & 447 & 303 & 750 \\
\hline
\end{tabular}

$P=0.202$, chi-square test with $2 d f$

Table 4 Numbers of male and female subjects with different alcohol-drinking habits

\begin{tabular}{llll}
\hline Alcohol-drinking habit $^{\mathrm{a}}$ & Male & Female & Total \\
\hline 0 & $75(17.0 \%)$ & $91(31.1 \%)$ & $166(22.6 \%)$ \\
1 & $131(29.7 \%)$ & $125(42.7 \%)$ & $256(34.9 \%)$ \\
2 & $235(53.3 \%)$ & $77(26.3 \%)$ & $312(42.5 \%)$ \\
Total & $441(100 \%)$ & $293(100 \%)$ & $734(100 \%)$
\end{tabular}

$P=1.83 \times 10^{-12}$, chi-square test with $2 d f$

a 0 nondrinker, 1 periodic drinker, 2 everyday drinker (almost every day)

positively correlated with gamma-GTP levels (Spearman's rank correlation test, $P<2.2 \times 10^{-16}, \quad r=0.408$, $n=752$ ), thereby showing that age is associated with
Table 5 Numbers of subjects with different alcohol-drinking habits and different genotypes at rs671

\begin{tabular}{lrrrc}
\hline \multicolumn{4}{c}{ Alcohol-drinking habit } & \\
\hline Entire sample $^{\mathrm{a}}$ & & & & \\
Genotype & 0 & 1 & 2 & Total \\
AA & 42 & 7 & 1 & 50 \\
AG & 63 & 100 & 89 & 252 \\
GG & 61 & 148 & 221 & 430 \\
Total & 166 & 255 & 311 & 732 \\
Male & & & & \\
Genotype & 0 & 1 & 2 & Total \\
AA & 28 & 6 & 1 & 35 \\
AG & 24 & 55 & 78 & 157 \\
GG & 23 & 69 & 155 & 247 \\
Total & 75 & 130 & 234 & 439 \\
Female & & & & \\
Genotype & 0 & 1 & 2 & Total \\
AA & 14 & 1 & 0 & 15 \\
AG & 39 & 45 & 11 & 95 \\
GG & 38 & 79 & 66 & 183 \\
Total & 91 & 125 & 77 & 293 \\
\hline
\end{tabular}

a $P<2.2 \times 10^{-16}$

b $P<2.2 \times 10^{-16}$

${ }^{\text {c }} P=1.75 \times 10^{-10}$ 
Fig. 4 Comparison of gammaglutamyltranspeptidase (gamma-GTP) levels between subjects with different alcoholdrinking habits. $N D$ nondrinker, $P D$ periodic drinker, $E D$ almost everyday drinker. a Male and female subjects were combined, or $\mathbf{b}$ male and $\mathbf{c}$ female subjects were analyzed separately
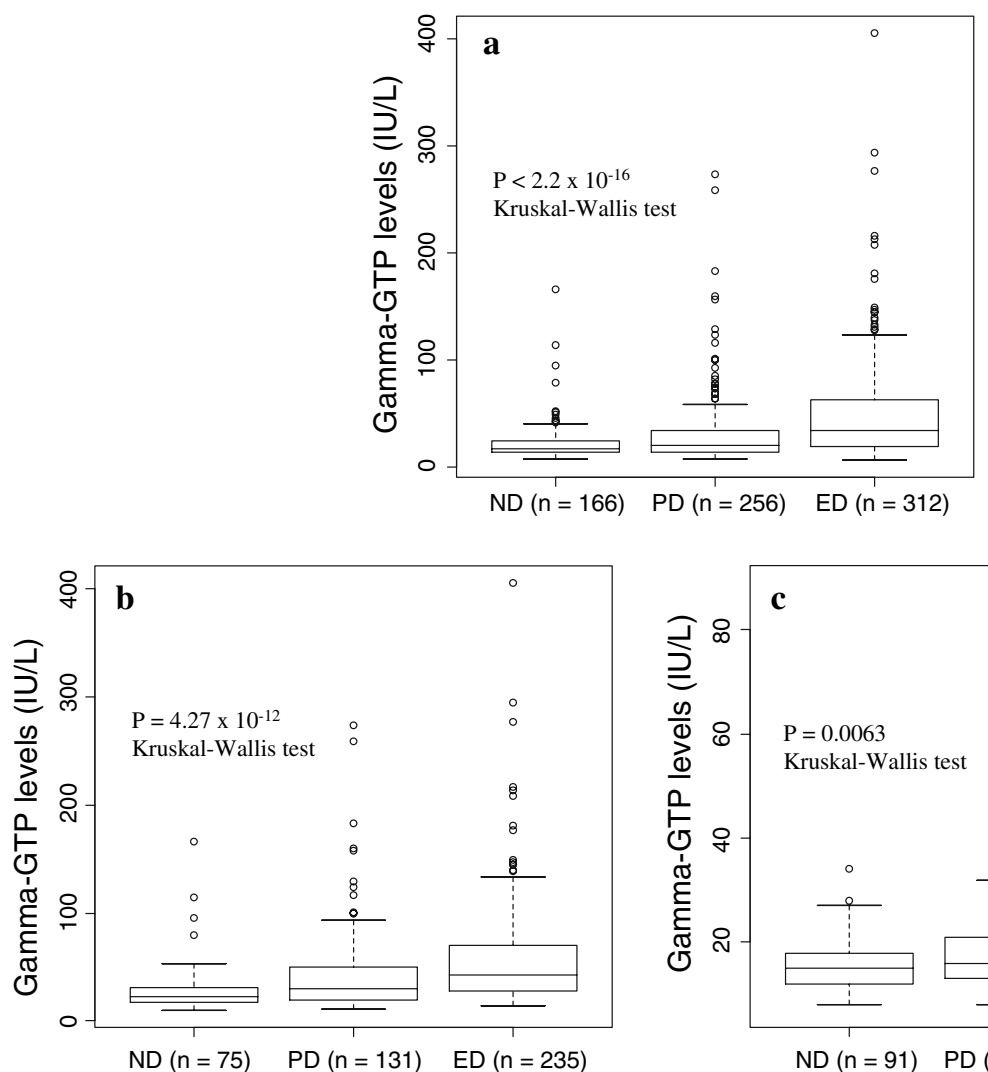

gamma-GTP level (Fig. 2h). The ages of male subjects ( $n=449$, mean 40.5 , SD 11.4) were significantly (MannWhitney $U$ test, $P=1.22 \times 10^{-9}$ ) higher than female subjects $(n=303$, mean 36.1, SD 11.5). Therefore, age and gender are associated with each other in our study (Fig. 2i). Then, we compared the ages between different rs671 genotypes. The ages in the three different genotypes were not significantly different (Kruskal-Wallis test, $P=0.5)$, thereby indicating that age and rs671 genotype are not associated with each other (Fig. 2j).

In summary, we have shown clear associations between all pairs of five factors in Fig. 2 except for rs671 genotype and gender and rs671 genotype and age. However, the structure of the relationship in Fig. 2 is very complicated and needs further investigation.

\section{Multiple regression analysis}

The first step to study the effects of various factors on a variable is multiple regression analysis. First, logistic multiple regression analysis was performed by setting alcohol-drinking habit as the criterion variable. In this binary-type analysis, alcohol-drinking habit was categorized as 0 (nondrinker or periodic drinker) or 1 (everyday drinker). The explanatory variables were age, gender, and rs671 genotype. Gamma-GTP level was not used because it does not likely affect the alcohol-drinking habit. Gender
Table 6 Logistic regression analysis for alcohol-drinking habit

\begin{tabular}{lcccl}
\hline & Estimate & SE & $z$ value & $P$ value \\
\hline Intercept & 0.949842 & 0.409261 & 2.321 & 0.0203 \\
Age & -0.043053 & 0.007448 & -5.78 & $7.46 \times 10^{-9}$ \\
Gender $^{\mathrm{a}}$ & 1.237978 & 0.179188 & 6.909 & $4.89 \times 10^{-12}$ \\
rs671 $^{\mathrm{b}}$ & -1.18164 & 0.152996 & -7.723 & $1.13 \times 10^{-14}$ \\
\hline
\end{tabular}

Logistic multiple regression analysis was performed with a criterion variable of alcohol-drinking habit (nondrinker or periodic drinker vs. everyday drinker) and explanatory variables of age, gender, and rs671 genotype. Estimated slope and its standard error (SE) of the regression line are shown along with $z$ value and $P$ value for the test of independence between an explanatory variable and the criterion variable. Note that a positive slope indicates that the explanatory variable increases the probability of nondrinker or periodic drinker but decreases the probability of everyday drinker

${ }^{\text {a }}$ Gender $($ male $=1$, female $=2$ )

b $\operatorname{rs} 671$ genotype $(\mathrm{AA}=-1, \mathrm{AG}=0, \mathrm{GG}=1)$

was coded as 1 (male) and 2 (female), and rs671 genotype was coded as -1 (AA), 0 (AG) and 1 (GG). Note that the explanatory variables age, gender, and rs671 genotype are theoretically independent from each other, although there was an association between age and gender (Fig. 2). Table 6 shows the results of the logistic multiple regression analysis. As shown, elder age, male gender, and $\mathrm{G}$ allele at rs671 significantly increase the probability of everyday 
Table 7 Linear multiple regression analysis

\begin{tabular}{|c|c|c|c|c|}
\hline & Estimate & SE & $t$ value & $P$ value \\
\hline \multicolumn{5}{|c|}{ Male and female subjects combined } \\
\hline Intercept & 3.401465 & 0.106906 & 31.817 & $<2.00 \times 10^{-16}$ \\
\hline Age & 0.015619 & 0.001799 & 8.683 & $<2.00 \times 10^{-1 \epsilon}$ \\
\hline Gender $^{\mathrm{a}}$ & -0.686244 & 0.043449 & -15.794 & $<2.00 \times 10^{-16}$ \\
\hline Alcohol $^{\mathrm{b}}$ & 0.144012 & 0.029096 & 4.95 & $9.25 \times 10^{-7}$ \\
\hline $\operatorname{rs} 671^{c}$ & 0.139902 & 0.035126 & 3.983 & $7.49 \times 10^{-5}$ \\
\hline \multicolumn{5}{|c|}{ Only male subjects } \\
\hline Intercept & 2.556348 & 0.116385 & 21.965 & $<2.00 \times 10^{-16}$ \\
\hline Age & 0.018261 & 0.002794 & 6.536 & $1.77 \times 10^{-10}$ \\
\hline Alcohol $^{\mathrm{b}}$ & 0.169143 & 0.044942 & 3.764 & 0.00019 \\
\hline rs67 $1^{c}$ & 0.175457 & 0.051388 & 3.414 & 0.000699 \\
\hline \multicolumn{5}{|c|}{ Only female subjects } \\
\hline Intercept & 2.265844 & 0.080371 & 28.192 & $<2.00 \times 10^{-16}$ \\
\hline Age & 0.0113 & 0.001977 & 5.717 & $2.71 \times 10^{-8}$ \\
\hline Alcohol $^{\mathrm{b}}$ & 0.083115 & 0.032039 & 2.594 & 0.00996 \\
\hline rs $671^{\mathrm{c}}$ & 0.098971 & 0.041197 & 2.402 & 0.01692 \\
\hline
\end{tabular}

Linear multiple regression analysis was performed. Criterion variable was $\log$ (gamma-GTP), and explanatory variables were age, gender, alcohol-drinking habit, and rs671 genotype when data from all subjects were analyzed. When data from either male or female subjects were analyzed, the criterion variable was the same but the explanatory variables were age, alcohol-drinking habit, and rs671 genotype

$S E$ standard error

${ }^{\mathrm{a}}$ Gender $($ male $=1$, female $=2)$

b Alcohol-drinking habit (nondrinker $=0$, periodic drinker $=1$, every-day drinker $=2$ )

${ }^{c}$ rs671 genotype $(\mathrm{AA}=-1, \mathrm{AG}=0, \mathrm{GG}=1)$

drinking. $P$ values were the lowest for the rs671 genotype, followed by gender and age. These results were as expected and the interpretation was straightforward.

In the next step, data was analyzed by linear multiple regression analysis with log gamma-GTP level as the criterion variable. In fact, log gamma-GTP level does not necessarily fit a normal distribution; however, it is much better than raw gamma-GTP level (data not shown). Explanatory variables were age, gender, alcohol-drinking habit, and rs671 genotype. Gender was coded as 1 (male) and 2 (female), whereas alcohol-drinking habit was coded as nondrinker (0), periodic drinker (1), and everyday drinker (2). The rs671 genotype was coded as -1 (AA), 0 (AG), and 1 (GG). There is a limitation in this analysis because explanatory variables are dependent on each other. Table 7 shows the results of this analysis. Although the results suggest that gender, age, alcohol-drinking habit, and rs671 genotype are associated with gamma-GTP level, the interpretation is not easy, because explanatory variables are associated with each other in a complicated manner (Fig. 2).
Subgroup analysis to examine the dependence of the association on other factors

Although investigation of the relationship between various factors as in Fig. 2 is not easy, a straightforward method is to examine the association in a rather homogeneous subgroup. One may stratify the entire sample into subgroups according to categorical variables until the subgroup populations become rather homogenous. However, the problem is that one cannot classify the sample according to a continuous variable and that the final subgroups may be too small for statistical analysis. By taking advantage of the large size of our sample, we attempted to perform association studies using subdivided samples. There were three categorical variables (gender, alcohol-drinking habit, and rs671 genotype). If we used all three categorical variables, we could divide the entire sample into 18 subgroups. However, the test of association using each of the 18 subgroups was not realistic. We may be able to ignore the effect of some factors because of the evidence of independence or very little dependence.

Association between rs671 genotype

and alcohol-drinking habit

We first wanted to show the association between alcoholdrinking habit and rs671 genotype independent of other factors. Since both age and gender were independent of rs671 genotype (Fig. 2c, j), these two factors could be ignored when the association between alcohol-drinking habit and rs671 genotype was considered. Gamma-GTP level can never be the cause of rs671 genotype and is not likely to be the cause of alcohol-drinking habit, and is also ignored. Therefore, the three factors, age, gender, and gamma-GTP level, could be ignored when we examined the independence of the association between rs671 genotype and alcohol-drinking habit. Since we previously showed the association between rs671 genotype and alcohol-drinking habit (Table 5; Fig. 2e), they are really associated with each other independent of gender and age. Then, we examined the association between rs671 genotype and alcohol-drinking habit in male and female subjects separately. Table 5 indicates that the association between rs671 genotype and alcohol-drinking habit was strong even when data for male and female subjects were analyzed separately. Thus, rs671 genotype was associated with alcohol-drinking habit independent of other factors.

Association between alcohol-drinking habit and gamma-GTP level

We next investigated whether the association between alcohol-drinking habit and gamma-GTP level (Fig. 2f) was 
Fig. 5 Comparison of gammaglutamyltranspeptidase (gamma-GTP) levels between subjects with different alcoholdrinking habits in the AG and GG genotypes at rs671. a Data from subjects with the genotype AG were analyzed. b Data from subjects with the genotype GG were analyzed. $N D$ nondrinker, $P D$ periodic drinker, $E D$ almost everyday drinker
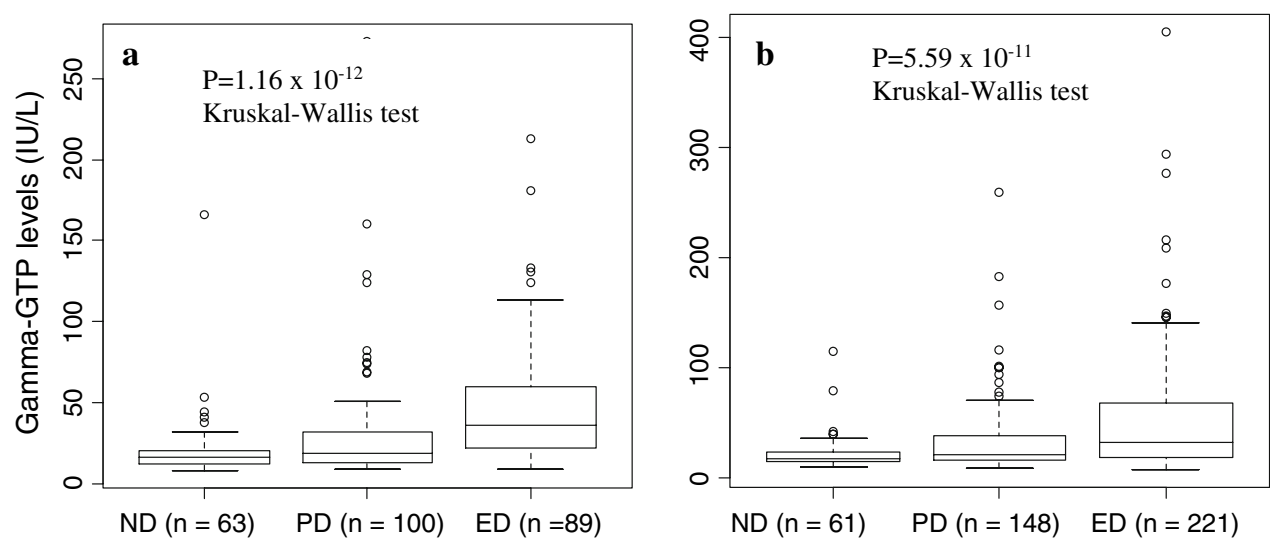

independent of other factors by subgroup analysis. Since gender and rs671 genotype were independent of each other (Fig. 2c), the effects of those factors on the association were studied separately. Figure $4 \mathrm{~b}$, c indicates that the association between alcohol-drinking habit and gammaGTP level was replicated in each gender. Next, we examined the association between alcohol-drinking habit and gamma-GTP level within each genotype. Comparison of gamma-GTP levels between the subjects with different alcohol-drinking habits in the group of AA genotype was not possible because of the small sample size. Therefore, we compared gamma-GTP levels between subjects with different alcohol-drinking habits in each of the groups of AG and GG genotypes (Fig. 5). Even among the subjects with the same genotypes (AG or GG), there were significant differences in gamma-GTP levels (Fig. 5a, b). In addition, we examined whether there was an association between alcohol-drinking habit and gamma-GTP level in male subjects with the GG genotype at rs671 $(n=265)$. Even in such a subgroup with the same gender and the same genotype, there was a difference in gamma-GTP level between different alcohol-drinking habits (Kruskal-Wallis test, $P=1.75 \times 10^{-5}, \mathrm{AA}=29, \mathrm{AG}=75, \mathrm{GG}=161$ ). Therefore, the association between alcohol-drinking habit and gamma-GTP level was independent of other factors.

\section{Association between rs671 genotype}

and gamma-GTP level

The association between gamma-GTP level and rs671 genotype was the first association detected by the association scan. We then examined whether the association between rs671 genotype and gamma-GTP level is independent of other factors. Since gender and age were independent of rs671 genotype (Fig. 2c, j), we examined whether the association was dependent on alcohol-drinking habit to show that the association was independent of other factors. However, since associations between gender and

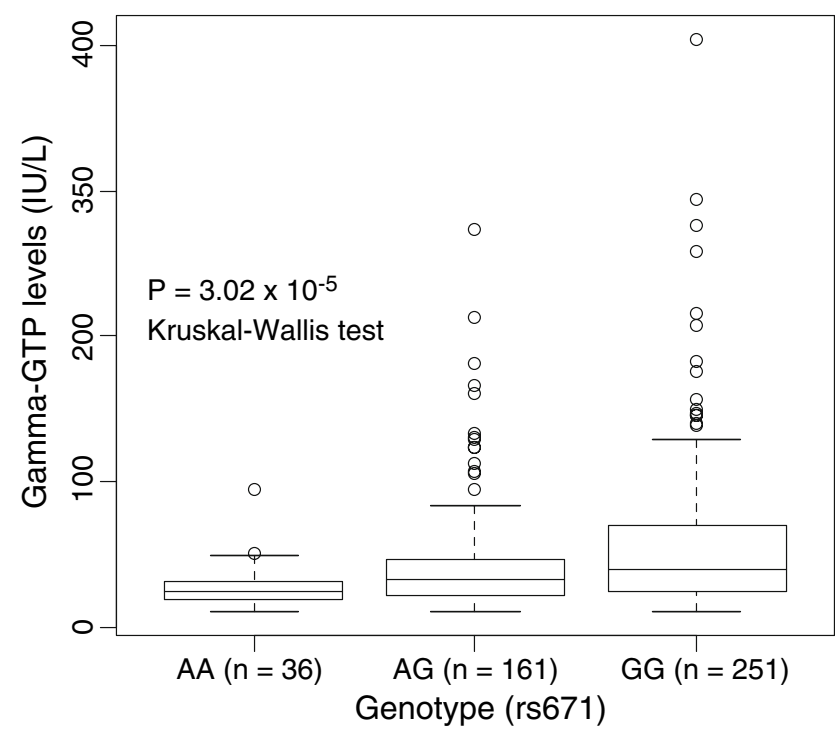

Fig. 6 Comparison of gamma-glutamyltranspeptidase (gamma-GTP) levels between different rs671 genotypes in male subjects

alcohol-drinking habit and between gender and gammaGTP level were very strong, we first examined the association between rs671 genotype and gamma-GTP level conditional on gender. In male subjects, the gamma-GTP levels between the three genotypes were significantly different as tested by the Kruskal-Wallis test $\left(P=3.02 \times 10^{-5}\right.$, Table 2, Fig. 6). When the heterozygotes (AG) were combined with either AA or GG homozygotes, the tests of the differences between two subgroups by Mann-Whitney test showed low $P$ values (AA vs. AG $+\mathrm{GG}, P=0.000275 ; \mathrm{AA}+\mathrm{AG}$ vs. $\mathrm{GG}$, $P=0.000146$ ) (Table 2). In general, $P$ values were lower when only male samples were examined than when the entire sample was used (Table 2). The results of the comparison of gamma-GTP levels in different genotypes in only the female samples are shown in Table 2 and Fig. 7. The Kruskal-Wallis test showed a $P$ value of $5.74 \times 10^{-5}$. Comparison between AA and AG + GG showed a $P$ value 


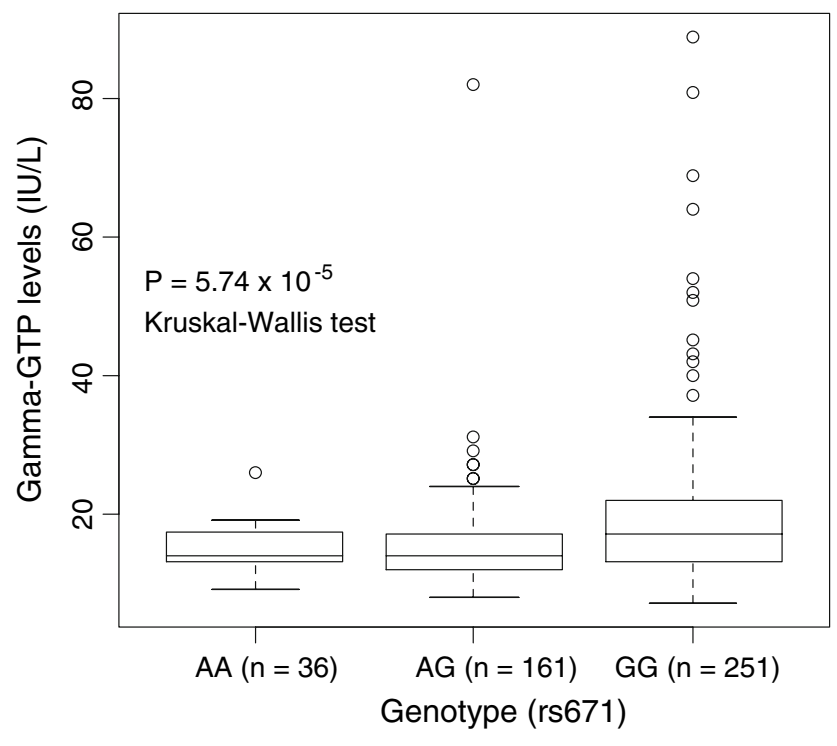

Fig. 7 Comparison of gamma-glutamyltranspeptidase (gamma-GTP) levels between different genotypes at rs671 in female subjects

of 0.3446, whereas comparison between $\mathrm{AA}+\mathrm{AG}$ and GG showed a $P$ value of $1.05 \times 10^{-5}$ (both by MannWhitney test).

Then the association between rs671 genotype and gamma-GTP level was examined conditional on alcoholdrinking habit. When the subjects were limited to nondrinkers $(n=166)$, the subjects with the AA genotype had higher gamma-GTP levels than those with AG or GG (Table $8, P=0.02$, Mann-Whitney test). When the same association was examined using all subjects, the presence of the $\mathrm{G}$ allele was positively associated with gamma-GTP level (Table 2; Fig. 1). The apparently paradoxical association between the rs671 genotype and gamma-GTP level disappeared when the analysis was performed in male and female subjects separately (Table 8 ). $P$ values obtained were all above 0.05 in male and female subjects (Table 8). The apparently paradoxical association between rs671 genotype and gamma-GTP level observed in the entire sample is explained by having both genders in the analysis. Thus, the proportion of AA genotype among nondrinker female subjects was $14 / 91=0.154$, whereas that among nondrinker male subjects was $28 / 75=0.373 \quad(P=$ 0.00022 , chi-square test). This is because the proportion of nondrinkers among subjects with AG or GG genotype was higher in female $(77 / 278=0.277)$ than in male subjects $(47 / 404=0.113) \quad\left(P=1.57 \times 10^{-7}, \quad\right.$ chi-square test $)$. Since gamma-GTP levels were higher in male than in female subjects (Fig. 3), even when the analyses were performed in nondrinkers (Table 8), the subjects with the AA genotype paradoxically showed higher gamma-GTP levels than the other genotypes when the analysis was done in nondrinkers (male and female subjects combined). The
Table 8 Association between gamma-glutamyltranspeptidase (gamma-GTP) levels and genotypes at rs671 in nondrinkers

\begin{tabular}{|c|c|c|c|c|c|c|}
\hline & \multirow[t]{2}{*}{ Number } & \multirow[t]{2}{*}{ Median } & \multirow[t]{2}{*}{ Mean } & \multirow[t]{2}{*}{ SD } & \multicolumn{2}{|c|}{ Statistical analysis } \\
\hline & & & & & Test & $\begin{array}{l}P \\
\text { value }\end{array}$ \\
\hline \multicolumn{7}{|c|}{ Entire sample } \\
\hline Total & 166 & 17 & 21.8 & 17.8 & & \\
\hline AA & 42 & 20 & 24.2 & 15.2 & & \\
\hline AG & 63 & 16 & 20.6 & 20.6 & $\begin{array}{l}\text { Kruskal- } \\
\text { Wallis }^{\mathrm{a}}\end{array}$ & 0.039 \\
\hline GG & 61 & 17 & 21.4 & 16.2 & & \\
\hline $\mathrm{AA}+\mathrm{AG}$ & 105 & 17 & 22 & 18.6 & MW (vs. GG) ${ }^{\mathrm{b}}$ & 0.89 \\
\hline $\mathrm{AG}+\mathrm{GG}$ & 124 & 17 & 21 & 18.5 & $\mathrm{MW}\left(\right.$ vs. AA) ${ }^{\mathrm{c}}$ & 0.02 \\
\hline \multicolumn{7}{|c|}{ Male subjects } \\
\hline Subtotal & 75 & 23 & 29.5 & 23.7 & & \\
\hline $\mathrm{AA}$ & 28 & 24.5 & 29 & 16.6 & & \\
\hline AG & 24 & 21.5 & 30.1 & 30.8 & $\begin{array}{l}\text { Kruskal- } \\
\text { Wallis }^{\mathrm{a}}\end{array}$ & 0.594 \\
\hline GG & 23 & 22 & 29.5 & 23.5 & & \\
\hline $\mathrm{AA}+\mathrm{AG}$ & 52 & 23.5 & 29.5 & 24 & MW (vs. GG) ${ }^{\mathrm{b}}$ & 0.573 \\
\hline $\mathrm{AG}+\mathrm{GG}$ & 47 & 22 & 29.9 & 27.2 & $\mathrm{MW}\left(\right.$ vs. AA) ${ }^{\mathrm{c}}$ & 0.311 \\
\hline \multicolumn{7}{|c|}{ Female subjects } \\
\hline Subtotal & 91 & 15 & 15.4 & 5 & & \\
\hline AA & 14 & 14 & 14.9 & 4.3 & & \\
\hline AG & 39 & 14 & 14.6 & 4.52 & $\begin{array}{l}\text { Kruskal- } \\
\text { Wallis }^{\mathrm{a}}\end{array}$ & 0.26 \\
\hline GG & 38 & 16 & 16.5 & 5.6 & & \\
\hline $\mathrm{AA}+\mathrm{AG}$ & 53 & 14 & 14.7 & 4.42 & MW (vs. GG) ${ }^{\mathrm{b}}$ & 0.11 \\
\hline $\mathrm{AG}+\mathrm{GG}$ & 77 & 15 & 15.5 & 5.13 & $\mathrm{MW}\left(\right.$ vs. AA) ${ }^{\mathrm{c}}$ & 0.8 \\
\hline
\end{tabular}

${ }^{a}$ Difference in gamma-GTP levels was tested between different genotypes (AA, AG, GG) by Kruskal-Wallis test

b Difference in gamma-GTP levels was tested by Mann-Whitney $U$ test between two groups, one composed of genotype GG and the other composed of the combination of two genotypes AA and AG

${ }^{c}$ Difference in gamma-GTP levels was tested by Mann-Whitney $U$ test between two groups, one composed of genotype AA and the other composed of the combination of two genotypes GG and AG

results of the analyses of the associations between rs671 genotypes and gamma-GTP levels in the subjects with a nondrinking habit suggest that the association between rs671 genotype and gamma-GTP level is dependent on alcohol-drinking habit. Therefore, the association between rs671 genotype and gamma-GTP level is not independent of other factors, and is an apparent association.

Association between gender and gamma-GTP level

Then we examined whether the association between gender and gamma-GTP level (Fig. 2b) was dependent on other factors. Gamma-GTP levels were significantly higher in male than in female subjects among nondrinkers 
Fig. 8 Correlation between age and $\log$ gamma-

glutamyltranspeptidase

(gamma-GTP) levels in male subjects was analyzed. a The correlation was analyzed only in male nondrinkers. b The correlation was analyzed only in male periodic drinkers. $\mathbf{c}$ The correlation was analyzed only in male everyday drinkers. The test of correlation was performed with the null hypothesis of correlation coefficient $(r)=0$
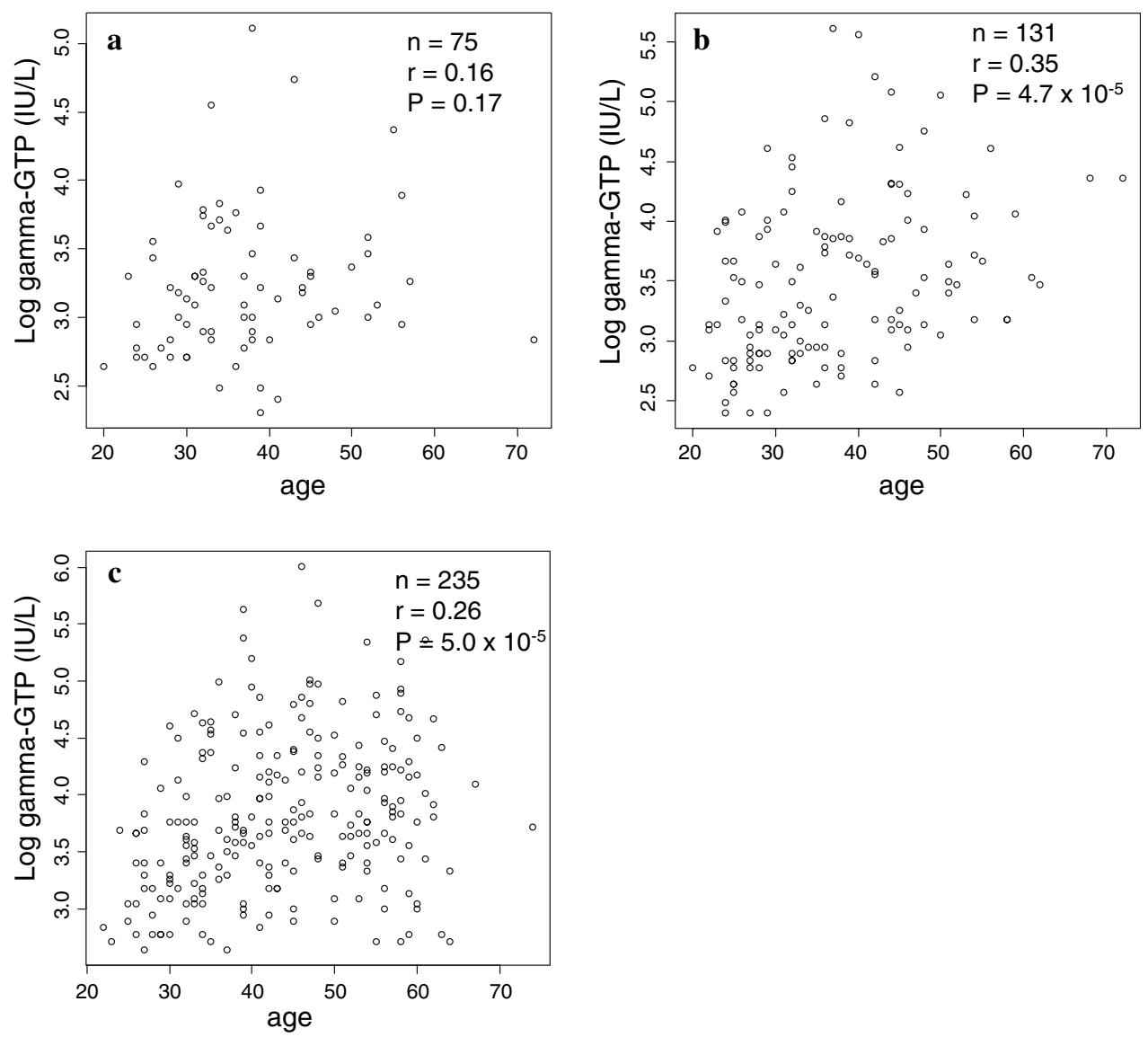

$\left(P=1.10 \times 10^{-12}\right.$, Mann-Whitney $U$ test, Fig. $\left.3 \mathrm{~b}\right)$, periodic drinkers $\left(P<2.22 \times 10^{-12}\right.$, Mann-Whitney $U$ test, Fig. 3c), and everyday drinkers $\left(P<2.22 \times 10^{-12}\right.$, Mann-Whitney $U$ test, Fig. 3d). These results indicate that gamma-GTP level is associated with gender independent of the alcohol-drinking habit. To show that the association between gamma-GTP level and gender is independent of other factors, the effect of age on the association should be examined. However, the test of correlation between age and gamma-GTP level in male and female subjects separately (but not stratified with the alcohol-drinking habit) has shown that the association was not significant either in male $(P=0.058$, Spearman's rank correlation test, $r=0.221)$ or in female subjects $(P=0.213$, Spearman's rank correlation test, $r=0.133$ ). Therefore, the association between gender and gamma-GTP level is not likely to depend greatly on age.

\section{Association between age and gamma-GTP level}

We examined whether the association between age and gamma-GTP level was independent of other factors. Figure 8 shows the relationship between age and log gammaGTP level in male subjects who were nondrinkers (Fig. 8 a), periodic drinkers (Fig. 8b), or everyday drinkers (Fig. 8c). There were significant positive correlations between age and log gamma-GTP level in both male periodic drinkers (Fig. 8 b, $P=4.7 \times 10^{-5}$ ) and male everyday drinkers (Fig. $8 \mathrm{c}, P=5.0 \times 10^{-5}$ ). Although there was a nonsignificant correlation between age and gamma-GTP level in male nondrinkers (Fig. 8a), it may reflect a small sample size $(n=75)$. When the associations were tested in nondrinkers (Fig. 9a, $P=0.021$ ), periodic drinkers (Fig. 9b, $P=6.75 \times 10^{-7}$ ), and everyday drinkers (Fig. 9c, $P=0.0015$ ) separately among female subjects, there were significant positive correlations.

Therefore, gamma-GTP level was positively correlated with age independent of gender and alcohol-drinking habit.

\section{Discussion}

A scan of 4,190 SNPs in 199 different genes using 38 different quantitative traits to search for associations between genotypes and phenotypes detected an association between rs671 genotypes and gamma-GTP levels. $P$ values, however, were far above the significance level after 
Fig. 9 Correlation between age and log gamma-

glutamyltranspeptidase (gamma-GTP) level in female subjects was analyzed. a The correlation was analyzed only in female nondrinkers. b The correlation was analyzed only in female periodic drinkers. c The correlation was analyzed only in female everyday drinkers. The test of correlation was performed with the null hypothesis of correlation coefficient $(r)=0$
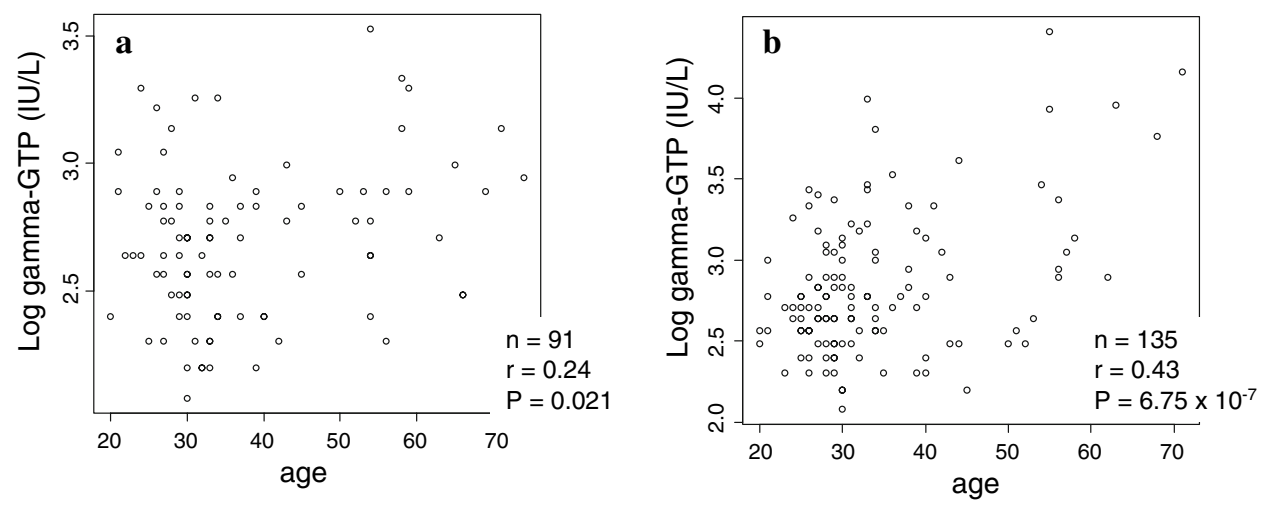

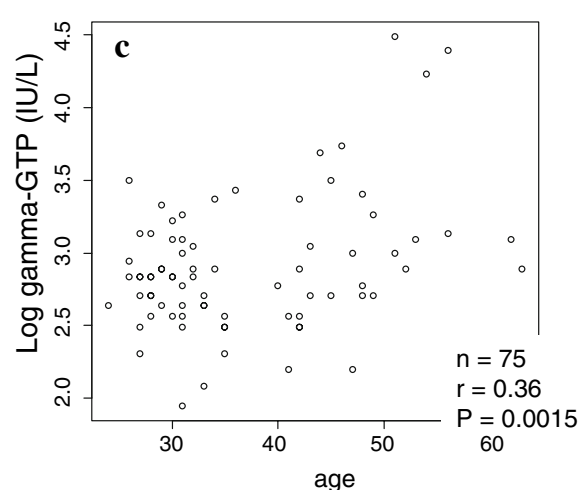

shown that all three explanatory variables are associated with the alcohol-drinking habit. Linear multiple regression analyses, by making gamma-GTP level as the criterion variable and age, gender, alcohol-drinking habit, and rs671 genotype as explanatory variables, have indicated that all explanatory variables are associated with gammaGTP level. However, more precise analyses were necessary to examine whether the associations are independent of other factors.

Subgroup analysis was very useful to examine whether an association of a pair of two variables was independent of the factors used for the stratification. In this way, the associations between rs671 genotype and alcohol-drinking habit, alcohol-drinking habit and gamma-GTP level, gender and gamma-GTP, and age and gamma-GTP were judged independent of other factors. Conversely, the association between rs671 genotype and gamma-GTP level was considered dependent on the alcohol-drinking habit. Although the direct associations between rs671 genotype and alcohol-drinking habit and between alcohol-drinking habit and gamma-GTP level are supported by functional evidence and previous reports, the associations between gamma-GTP level and gender and gamma-GTP level and age are of interest. The associations between gamma-GTP and age (Puukka et al. 2006) and gamma-GTP and gender (Stewart et al. 2007) have been previously reported.

The associations between genotypes and multiple phenotypes are often very complicated. Previous studies have 
shown the association between $A L D H 2$ genotypes and hypertension (Tsuritani et al. 1995). In addition, gammaGTP levels have been reported to be associated with hypertension (Takagi et al. 2001; Shankar and Li 2007), cardiovascular event (Fraser et al. 2007b; Patel et al. 2007; Yang et al. 2007; Hozawa et al. 2007; Turgut et al. 2006; Lee et al. 2006), type 2 diabetes (Doi et al. 2007; Fraser et al. 2007a; Lim et al. 2007), obesity (Lim et al. 2007), adiponectin (Kim et al. 2007), hyperlipidemia (Lippi et al. 2007), and metabolic syndrome (Andre et al. 2007; Lee et al. 2007). Although most of these associations are thought to be indirect and dependent on other factors such as alcohol-drinking habit, careful analyses of the data from the statistical viewpoint are necessary.

Associations between genetic and environmental factors will become a focus of medical and biological studies, since recent genome-wide association studies are expected to identify many association candidates (Ozaki et al. 2002; Suzuki et al. 2003; Kochi et al. 2005; The Wellcome Trust Case Control Consortium 2007). Our study reported here shows that (1) a large sample size combined with a replication study is necessary to overcome the multiplecomparison problem, and (2) subgroup analysis along with logistic and linear multiple regression analysis may be useful to dissect the complicated relationship.

Acknowledgments This research was supported by the Health and Labour Sciences Research Grants from Research on Human Genome tailor-made by the Ministry of Health, Labour and Welfare of Japan. We thank Ms. Aki Nakano for editorial assistance.

\section{References}

Andre P, Balkau B, Vol S, Charles MA, Eschwege E (2007) Gammaglutamyltransferase activity and development of the metabolic syndrome (International Diabetes Federation Definition) in middle-aged men and women: Data from the Epidemiological Study on the Insulin Resistance Syndrome (DESIR) cohort. Diabetes Care 30:2355-2361

Boone DJ, Tietz NW, Weinstock A (1977) Significance of gammaglutamyl transferase (GGT) activity measurements in alcoholinduced hepatic injury. Ann Clin Lab Sci 7:25-28

Chehab FF, Johnson J, Louie E, Goossens M, Kawasaki E, Erlich H (1991) A dimorphic 4-bp repeat in the cystic fibrosis gene is in absolute linkage disequilibrium with the delta F508 mutation: implications for prenatal diagnosis and mutation origin. Am J Hum Genet 48:223-226

Crabb DW, Edenberg HJ, Bosron WF, Li TK (1989) Genotypes for aldehyde dehydrogenase deficiency and alcohol sensitivity. The inactive ALDH2(2) allele is dominant. J Clin Invest 83:314316

Doi Y, Kubo M, Yonemoto K, Ninomiya T, Iwase M, Tanizaki Y, Shikata K, Iida M, Kiyohara Y (2007) Liver enzymes as a predictor for incident diabetes in a Japanese population: the Hisayama study. Obesity 15:1841-1850

Feldman MW, Franklin I, Thomson GJ (1974) Selection in complex genetic systems. I. The symmetric equilibria of the three-locus symmetric viability model. Genetics 76:135-162
Fraser A, Ebrahim S, Smith GD, Lawlor DA (2007a) A comparison of associations of alanine aminotransferase and gamma-glutamyltransferase with fasting glucose, fasting insulin, and glycated hemoglobin in women with and without diabetes. Hepatology 46:158-165

Fraser A, Harris R, Sattar N, Ebrahim S, Smith GD, Lawlor DA (2007b) Gamma-glutamyltransferase is associated with incident vascular events independently of alcohol intake: analysis of the British Women's Heart and Health Study and Meta-analysis. Arterioscler Thromb Vasc Biol 27:2729-2735

Goedde HW, Agarwal DP, Harada S, Rothhammer F, Whittaker JO, Lisker R (1986) Aldehyde dehydrogenase polymorphism in North American, South American, and Mexican Indian populations. Am J Hum Genet 38:395-399

Goedde HW, Agarwal DP, Fritze G, Meier-Tackmann D, Singh S, Beckmann G, Bhatia K, Chen LZ, Fang B, Lisker R et al (1992) Distribution of $\mathrm{ADH} 2$ and $\mathrm{ALDH} 2$ genotypes in different populations. Hum Genet 88:344-346

Hozawa A, Okamura T, Kadowaki T, Murakami Y, Nakamura K, Hayakawa T, Kita Y, Nakamura Y, Okayama A, Ueshima H (2007) NIPPON DATA90 research group. Gamma-Glutamyltransferase predicts cardiovascular death among Japanese women. Atherosclerosis 194:498-504

Impraim C, Wang G, Yoshida A (1982) Structural mutation in a major human aldehyde dehydrogenase gene results in loss of enzyme activity. Am J Hum Genet 34:837-841

Ito T, Inoue E, Kamatani N (2004) Association test algorithm between a qualitative phenotype and a haplotype or haplotype set using simultaneous estimation of haplotype frequencies, diplotype configurations and diplotype-based penetrances. Genetics 168: 2339-2348

Kamatani N, Sekine A, Kitamoto T, Iida A, Saito S, Kogame A, Inoue E, Kawamoto M, Harigai M, Nakamura Y (2004) Large-scale single-nucleotide polymorphism (SNP) and haplotype analyses, using dense SNP Maps, of 199 drug-related genes in 752 subjects: the analysis of the association between uncommon SNPs within haplotype blocks and the haplotypes constructed with haplotype-tagging SNPs. Am J Hum Genet 75:190-203

Kasperczyk A, DiMartino NA, Krontiris TG (1990) Minisatellite allele diversification: the origin of rare alleles at the HRAS1 locus. Am J Hum Genet 47:854-859

Kim JA, Kim SM, Yoon D (2007) The relationship between gammaglutamyltransferase and adiponectin in nonalcoholic women. Metabolism 56:578-582

Kochi Y et al (2005) A functional variant in FCRL3, encoding Fc receptor-like 3 , is associated with rheumatoid arthritis and several autoimmunities. Nat Genet 37:478-485

Lee DH, Silventoinen K, Hu G, Jacobs DR Jr, Jousilahti P, Sundvall J,Tuomilehto J (2006) Serum gamma-glutamyltransferase predicts non-fatal myocardial infarction and fatal coronary heart disease among 28,838 middle-aged men and women. Eur Heart J 27:2170-2176

Lee DS, Evans JC, Robins SJ, Wilson PW, Albano I, Fox CS, Wang TJ, Benjamin EJ, D'Agostino RB, Vasan RS (2007) Gamma glutamyl transferase and metabolic syndrome, cardiovascular disease, and mortality risk: the Framingham heart study. Arterioscler Thromb Vasc Biol 27:127-133

Lim JS, Lee DH, Park JY, Jin SH, Jacobs DR Jr (2007) A strong interaction between serum gamma-glutamyltransferase and obesity on the risk of prevalent type 2 diabetes: results from the third national health and nutrition examination survey. Clin Chem 53:1092-1098

Lippi G, Targher G, Montagnana M, Salvagno GL, Guidi GC (2007) Relationship between gamma-glutamyltransferase, lipids and lipoprotein(a) in the general population. Clin Chim Acta 384:163-166 
Ohnishi Y, Tanaka T, Ozaki K, Yamada R, Suzuki H, Nakamura Y (2001) A high-throughput SNP typing system for genome-wide association studies. J Hum Genet 46: 471-477

Ozaki K et al (2002) Functional SNPs in the lymphotoxin-alpha gene that are associated with susceptibility to myocardial infarction. Nat Genet 32:650-654

Patel DA, Srinivasan SR, Xu JH, Chen W, Berenson GS (2007) Persistent elevation of liver function enzymes within the reference range is associated with increased cardiovascular risk in young adults: the Bogalusa heart study. Metabolism 56:792-798

Puukka K, Hietala J, Koivisto H, Anttila P, Bloigu R, Niemela O (2006) Age-related changes on serum ggt activity and the assessment of ethanol intake. Alcohol Alcohol 41:522-527

Rosalki SB (1973) Letter: screening test for alcoholism. Lancet 7833:843

Rosalki SB (1974) Letter: gamma-glutamyl transpeptidase in alcoholism. Am J Clin Pathol 62:579-580

Shankar A, Li J (2007) Association between serum gamma-glutamyltransferase level and prehypertension among US adults. Circ J 71:1567-1572

Shibata K, Ito T, Kitamura Y, Iwasaki N, Tanaka H, Kamatani N (2004) Simultaneous estimation of haplotype frequencies and quantitative trait parameters: applications to the test of association between phenotype and diplotype configuration. Genetics 168:525-539

Shibuya A, Yasunami M, Yoshida A (1989) Genotype of alcohol dehydrogenase and aldehyde dehydrogenase loci in Japanese alcohol flushers and nonflushers. Hum Genet 82:14-16

Spencer-Peet J, Wood DC, Glatt MM, Wiseman SM (1975) Urinary D-glucaric acid excretion and serum gamma-glutamyl transpeptidase activity in alcoholism. Br J Addict Alcohol Other Drugs 70:359-364
Stewart SH, Connors GJ, Hutson A (2007) Ethnicity and gammaglutamyltransferase in men and women with alcohol use disorders. Alcohol Alcohol 42:24-27

Suzuki A et al (2003) Functional haplotypes of PADI4, encoding citrullinating enzyme peptidylarginine deiminase 4 , are associated with rheumatoid arthritis. Nat Genet 34:395-402

Takagi S, Baba S, Iwai N, Fukuda M, Katsuya T, Higaki J, Mannami T, Ogata J, Goto Y, Ogihara T (2001) The aldehyde dehydrogenase 2 gene is a risk factor for hypertension in Japanese but does not alter the sensitivity to pressor effects of alcohol: the Suita study. Hypertens Res 24:365-370

Teschke R, Brand A, Strohmeyer G (1977) Induction of hepatic microsomal gamma-glutamyltransferase activity following chronic alcohol consumption. Biochem Biophys Res Commun 175:718-724

The Wellcome Trust Case Control Consortium (2007) Genome-wide association study of 14,000 cases of seven common diseases and 3,000 shared controls. Nature 447:661-678

Tsuritani I, Ikai E, Date T, Suzuki Y, Ishizaki M, Yamada Y (1995) Polymorphism in ALDH2-genotype in Japanese men and the alcohol-blood pressure relationship. Am J Hypertens 8:10531059

Turgut O, Yilmaz A, Yalta K, Karadas F, Birhan Yilmaz M (2006) gamma-Glutamyltransferase is a promising biomarker for cardiovascular risk. Med Hypotheses 67:1060-1064

Walter MA, Cox DW (1988) Analysis of genetic variation reveals human immunoglobulin VH-region gene organization. Am J Hum Genet 42:446-451

Yang JJ, Shiwaku K, Nabika T, Masuda J, Kobayashi S (2007) High frequency of cardiovascular risk factors in overweight adult Japanese subjects. Arch Med Res 38:337-344 